\title{
An Alternative Choice in Heighting
}

\author{
O. G. Omogunloye*, M. O. Yaqub, B. M. Ojegbile, J. O. Odumosu and O. G. Ajayi \\ University of Lagos, Nigeria, Surveying and Geoinformatics \\ ${ }^{*}$ Corresponding author: oomogunloye@unilag.edu.ng
}

\begin{abstract}
Comparison of orthometric heights obtained from the combination of GPS/Levelling survey method with that obtained from Light Detection Ranging (LIDAR), Shuttle Radar Topography Mission (SRTM), and Advanced Spaceborne Thermal Emission and Reflection Radiometer (ASTER GDEM) data is an area of research which is of great interest to Geomaticians. This area of research makes possible the discovery of other suitable methods of determining orthometric height which can be selected for use, depending on the region, extent and nature of the terrain where the project is to be executed.

The X, Y, Z coordinates and the geoidal heights for all the existing controls within university of Lagos were determined using the GPS/ Levelling survey method, the required orthometric height $(\mathrm{H})$ was then obtained as the differences between ellipsoidal and geoidal heights. Extracting orthometric heights for the X and Y coordinates of observed control points, overlaid on each of LIDAR, SRTM and ASTER GDEM, required the use of spatial analysis tool in an ArcMap environment. From the profile plot (Figure 8) of all the orthometric heights, the heights relationship was easily established. From the descriptive statistics test (Table 4), the one way ANOVA test at 1\% and 5\% level of significance (Table 7), the number of points in other methods whose orthometric height is closed to that of Levelling/GPS method (Table 6) and correlation test on the various orthometric heights obtained (Table 8), it is obvious that all the applied methods operates at different spatial resolutions. Of all the four methods, GPS/Levelling method, was the most reliable and most accurate method followed by LIDAR method, then by ASTER GDEM method and SRTM has the least. In a nutshell, orthometric heights generated by method of LIDAR are very close to that generated by GPS/Levelling method at several stations, thus method of LIDAR was considered as the most suitable alternative to GPS/Levelling method, whenever the use of the later method cannot be easily accomplished.
\end{abstract}

Keywords: Orthometric heights; GPS levelling; LIDAR; SRTM; ASTER GDEM.

\section{Introduction}

The quest for several methods of determining orthometric heights truly call for research in this area; the Leveling/GPS survey method which is considered the most accurate method may not be suitable in some region as a result of which other methods such as the use of LIDAR, SRTM and ASTER GDEM (Leon et al., 2014; Rodriguez, et al., 2005) can actually come into play. Generation of orthometric heights from other methods required the overlay of sample points (X and Y coordinates of observed control stations downloaded from GPS) on each of the data in an arc map environment. The use of spatial analysis tool in an arc map environment enable easy extraction of values from points overlaid, these values are the orthometric heights required from each of the data. Orthometric height $(\mathrm{H})$, (height above the geoid) relates to the Earth's physical surface and are needed by most of the Geomatics Engineering, surveying, geodetic and geophysics applications. Though the development of Global Positioning Systems (GPS) techniques has been helpful in height determination, our aim at replacing the classical Levelling with GPS surveys may not be fruitful, since the main 
problem with GPS technique is that its height refers to a reference ellipsoid approximating the true shape of the Earth but not the geoid or MSL.

\subsection{Area of Study}

The study area is the entire University of Lagos. It has a total area of 146.815 hectares. Figure 1 shows the map of the University of Lagos. It is located on the western part of Lagos metropolis in Lagos Mainland Local Government Area of Lagos State. The University is geographically located between Northing's 721000N and 718500N, and Easting 542000E and $545000 \mathrm{E}$.



Figure 1: Map of University of Lagos.

\subsection{Aim of the Study}

To determine and compare the orthometric heights obtained for all the existing controls within University of Lagos, using GPS/Levelling survey method, LIDAR, SRTM and ASTER GDEM data, as well as their ranking. 


\subsection{Objectives of the Study}

- To obtain the ellipsoidal heights of all existing controls within University of Lagos using the method of GPS.

- To compute the geoidal heights of all the existing controls within the University using levelling survey method.

- To compute the orthometric heights of all existing controls within University of Lagos using (i) and (ii).

- To extract the corresponding orthometric heights of all the controls from LIDAR data, SRTM and ASTER GDEM.

- To rank the various methods of height determination used based on their accuracy, reliability and efficiency.

- To determine which of the methods of generating orthometric heights from (iv) are suitable in our study area, as an alternative to the Leveling/GPS survey method.

- To plot the profile of the various orthometric heights obtained, so as to know how they relates to one another.

\subsection{Scope of the Study}

The scope of the project is as follows:

- To use dual frequency differential GPS to obtain X, Y, Z coordinates of all existing controls within University of Lagos.

- To carry out the Levelling survey across all existing controls within University of Lagos.

- To obtained the orthometric heights of all the existing control from (i) and (ii) using the relation $H=h-N$.

- To produce a digital drawing of all the coordinated controls using AutoCAD 2013.

- To extract the corresponding orthometric heights of all the coordinated controls from LIDAR data, SRTM data and ASTER GDEM data, through overlay of sample points (In ArcMap).

- To produce the profile drawing of all the orthometric heights obtained from the various methods using Excel.

\subsection{Significance of the Study}

This study is however to provide an enhanced alternative choice in heighting when and where GPS/Levelling (Colombo, 1986 \& 1989) survey method is not easily practicable or necessary, especially when a geodetic accuracy is not of immense requirement. The study tries to computes the orthometric heights of all existing control points within University of Lagos using several methods such as GPS/Levelling methods, LIDAR, SRTM and ASTER GDEM methods, which may be required in surveying, engineering, geodetics and geophysical applications. 


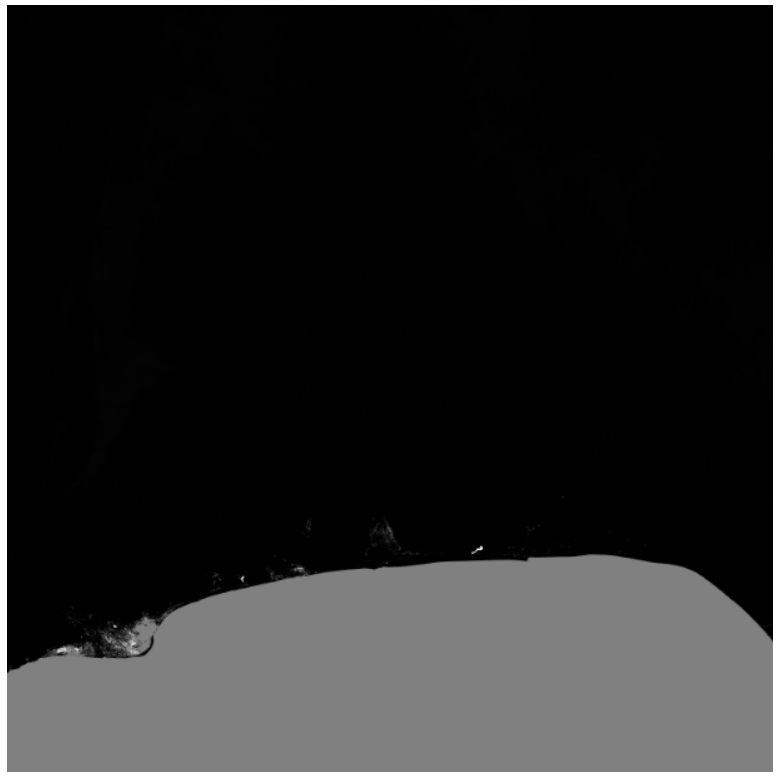

Figure 2: SRTM Data (from http://SRTM.cgiar.csi.org)

\subsection{Research Questions}

The research proffers answer to the following questions:

- Which of the other methods of generating orthometric heights can be use as an alternative to Levelling survey method?

- Under what conditions do the various methods apply?

- What was the order of ranking of the various methods?

- What is the justification for the alternative choice to a given project?

\subsection{Limitation of the Research Work}

- The various methods applied operates at different spatial resolution

- The epoch at which the various methods operate are not the same

\section{LIDAR, SRTM, and ASTER GDEM Data}

The Digital Elevation Model (DEM) acquired for the research was a $90 \mathrm{~m}$ spatial resolution SRTM version 4.1 which corresponds to the study areas. It was downloaded from the site http://SRTM.csi.cgiar.org. It is available in 5 degree $\times 5$ degree tiles, in Geographic decimal degrees projection, with WGS84 (World geodetic system of 1984) horizontal datum and EGM96 (Earth gravity model) vertical datum, (Colombo, 1986). This version 4.1 was chosen for this research because it has been updated, and then released after using sophisticated interpolation and hole-filling algorithms (www.cgiar-csi.org). Figure 2 shows a typical SRTM data, (Rodriguez, et.al, 2005). 


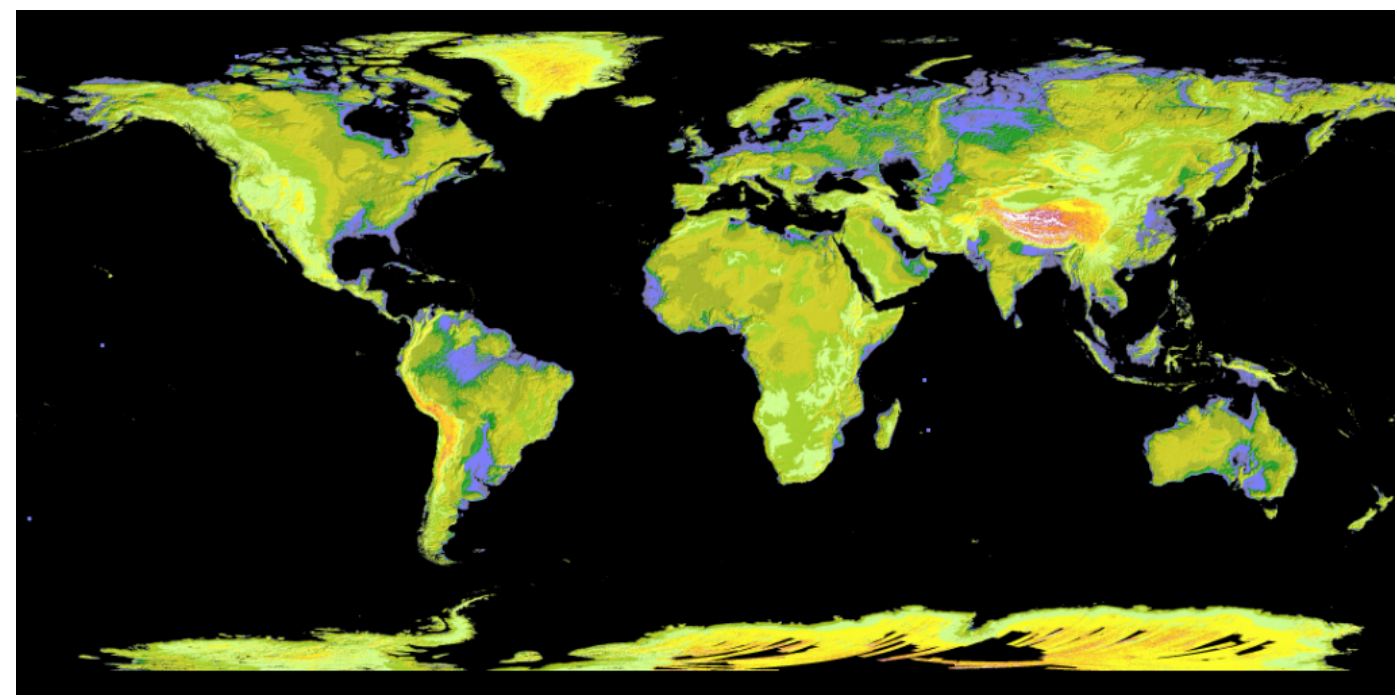

Figure 3: ASTER GDEM Data from the site http://earthdata.nasa.gov

ASTER DEM (Digital elevation model) version 2 corresponding to the same study areas was downloaded from https://earthdata.nasa.gov, which are posted on a 1 arc-second (approximately $30-\mathrm{m}$ at the equator) grid, distributed as $1^{\circ} \times 1^{\circ}$ tiles and referenced to the World Geodetic System, 1984 (WGS84)/ Earth Gravitational Model 1996 (EGM96) geoid. A 5-m overall bias observed on GDEM 1.0 was removed in this version 2 by ASTER GDEM VALIDATION TEAM, 2009. Figure 3 shows a typical ASTER GDEM data.

LIDAR is a direct acquisition system, in which obtaining ground points is performed in a global reference system, through the use of GPS and inertial systems that control the position and orientation of the scanner during the capture of information. The determination of the coordinates of points is obtained from the position and orientation of the scanner at every moment and the distance observed at each point. Through this data capture system, (Becker, 1986), it is possible to obtain a set of points whose reference system will be WGS84. Figure 4 shows a typical LIDAR data.

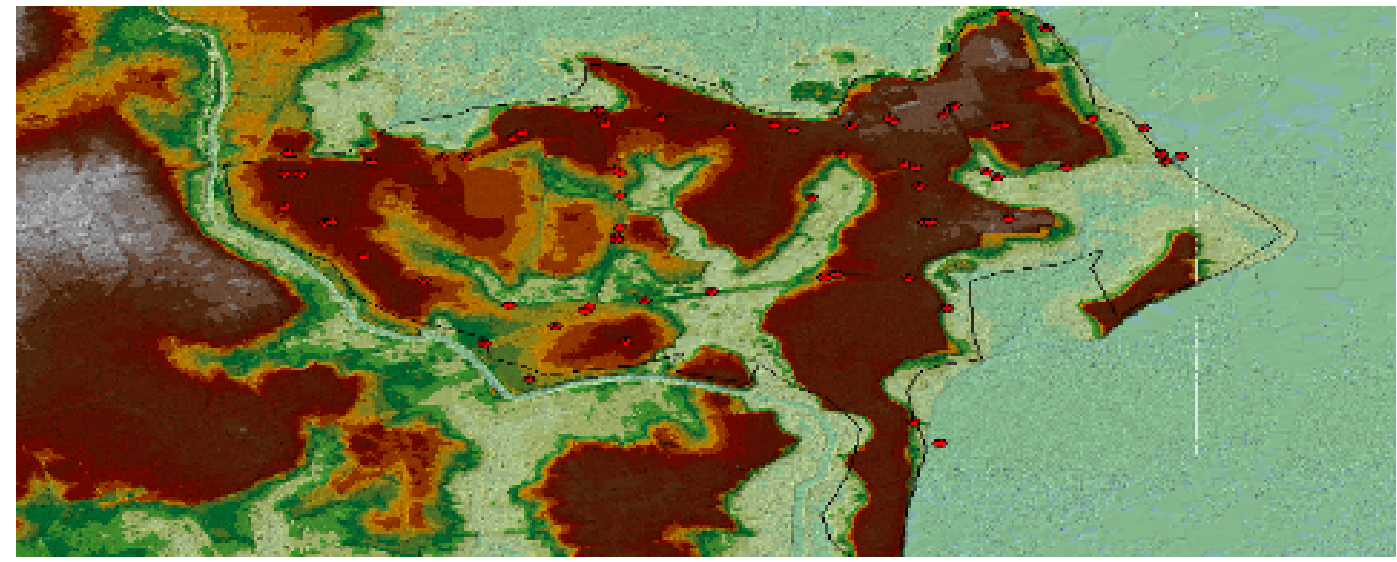

Figure 4: LIDAR Data Obtained From the Surveyor General Office. 


\subsection{Data Set and Field Observation}

From the reconnaissance survey carried out, Unilag 1 control point was selected as the base station for our GPS survey; all other control points serve as the rover stations, (Colombo, 1986).

Having carried out the GPS Survey as stated above, the X, Y, and Z Coordinates of all the observed control points was then downloaded from the GPS via downloading cable, (Colombo, 1989). With Unilag 1 as the reference station, we carried out the Levelling survey across all the existing control points within University of Lagos. It was a closed loop Levelling network that was run across all the controls. The data acquired from the Levelling network was then reduced using the height of collimation method (see later), to obtain the geoidal heights for all the observed control points.

Having known the geoidal heights, the required orthometric heights were then obtained using the relation, (Heister et al., 1999. Hofmann-Wellenhof et al., 2008).

$$
H=h-N
$$

All necessary procedures and precautions that ensure the utmost accuracy of the survey were used. The GPS survey was connected to national grid and the Level survey connected to the Mean Sea level.

\subsection{GPS Downloaded Coordinates for Observed Control Points}

In this research work, the GPS observation has been carried out on 44 control points within the University of Lagos, to obtained accurate three dimensional coordinates of all the observed controls points (with a precision of 1 part per million). Sample Table 1 shows the downloaded coordinates of control points observed, (Colombo, 1989).

Table 1: Coordinates of Observed Controls and ED015 their Geoidal Heights

\begin{tabular}{|l|c|c|c|c|}
\hline \multirow{2}{*}{ Station ID } & \multicolumn{4}{c|}{ orthometric heights $(\mathrm{m})$} \\
& Levelling/GPS & \multicolumn{1}{c|}{ LIDAR } & \multicolumn{1}{c|}{ SRTM } & \multicolumn{1}{c|}{ ASTER GDEM } \\
\hline YTT28/186 & 1.234 & 1.496 & 12.000 & 6.795 \\
\hline ED015 & 4.970 & 4.556 & 11.000 & 5.656 \\
\hline SD15S & 4.560 & 3.750 & 7.000 & 6.353 \\
\hline DOS14S & 4.340 & 4.445 & 10.000 & 5.545 \\
\hline GME03 & 5.035 & 6.779 & 11.000 & 1.415 \\
\hline CR02 & 3.646 & 3.014 & 6.000 & 3.431 \\
\hline MEGA01 & 4.547 & 4.086 & 3.000 & 6.041 \\
\hline SGDP016 & 4.511 & 5.813 & 4.000 & 3.379 \\
\hline SGEN10 & 4.465 & 4.375 & 10.000 & 3.923 \\
\hline SGEN11 & 3.795 & 4.602 & 9.000 & 4.899 \\
\hline USL01 & 5.902 & 6.539 & 3.000 & 6.879 \\
\hline
\end{tabular}


O. G. Omogunloye et al.: An Alternative Choice in heighting

Table 2: Orthometric heights extracted from various methods.

\begin{tabular}{|l|c|c|c|c|}
\hline \multirow{2}{*}{ Station ID } & \multicolumn{4}{c}{ Orthometric heights $(\mathrm{m})$} \\
& Levelling/GPS & \multicolumn{1}{c|}{ LIDAR } & \multicolumn{1}{c|}{ SRTM } & \multicolumn{1}{c|}{ ASTER GDEM } \\
\hline UNILAG1 & 4.859 & 3.976 & 0.000 & 3.693 \\
\hline UNILAG2 & 4.030 & 3.870 & 0.000 & 7.177 \\
\hline DSG08 & 2.522 & 1.258 & 10.000 & 1.358 \\
\hline MEGA05 & 3.525 & 2.843 & 0.000 & 4.931 \\
\hline MEGA06 & 2.127 & 1.856 & 10.000 & 1.995 \\
\hline MEGA03 & 3.042 & 3.648 & 11.000 & 4.729 \\
\hline PGS09 & 3.597 & 3.949 & 10.000 & 4.414 \\
\hline GME02 & 3.850 & 4.077 & 11.000 & 6.848 \\
\hline MEGA04 & 4.470 & 4.040 & 11.000 & 4.995 \\
\hline GME06 & 5.272 & 6.705 & 8.000 & 3.740 \\
\hline GME05 & 5.030 & 5.523 & 12.000 & 6.985 \\
\hline GME04 & 3.115 & 3.107 & 10.000 & 5.553 \\
\hline PGD84/2 & 3.698 & 4.429 & 5.000 & 4.585 \\
\hline MEGA07 & 1.779 & 1.608 & 9.000 & 6.528 \\
\hline PGD81/1 & 3.968 & 4.574 & 11.000 & 5.602 \\
\hline ED009 & 3.628 & 3.329 & 8.000 & 5.355 \\
\hline MEGA09 & 4.172 & 3.729 & 7.000 & 5.849 \\
\hline DOS03 & 4.488 & 5.233 & 9.000 & 5.433 \\
\hline MEGA10 & 4.281 & 4.795 & 9.000 & 4.514 \\
\hline MEGA11 & 3.970 & 3.139 & 10.000 & 4.314 \\
\hline XST347 & 3.908 & 3.723 & 8.000 & 7.568 \\
\hline MEGA02 & 4.694 & 5.461 & 8.000 & 0.843 \\
\hline CR08 & 3.619 & 3.386 & 8.000 & 3.386 \\
\hline SD14S & 5.490 & 4.214 & 6.000 & 4.169 \\
\hline CR07 & 3.519 & 4.819 & 6.000 & 4.471 \\
\hline CR06 & 3.630 & 3.393 & 6.000 & 4.395 \\
\hline CR05 & 3.639 & 3.061 & 7.000 & 3.461 \\
\hline CR03 & 3.944 & 2.931 & 5.000 & 3.132 \\
\hline ED011 & 3.915 & 3.573 & 5.000 & 2.973 \\
\hline DSG07 & 1.666 & 1.258 & 10.000 & 1.258 \\
\hline CGG/SP & 3.787 & 3.078 & 8.000 & 6.528 \\
\hline XST347AZ & 5.873 & 4.862 & 8.000 & 7.553 \\
\hline CBLM3 & 4.058 & 4.501 & 7.000 & 4.701 \\
\hline & & & & \\
\hline
\end{tabular}

\subsection{Levelling Data for Observed Control Points and its Reduction}

Instead of writing the readings in a sketch and giving suitable descriptions, the whole process of levelling was systematically shown in a level book and reduced levels of different points found out (Heister, et, al., 1999). There are two methods of reducing levels. (i) Rise and fall method, and (ii) Height of collimation method, complete bookings and reductions in the height of collimation method. 


\section{Height of Collimation Method}

The height of collimation is obtained by adding the back sight staff reading to known station and the reduced level (R.L) of same known point on which the staff stands. R.L of all the other points are obtained by subtracting the staff reading from the height of collimation. When the instrument is changed a new height of collimation is obtained by again adding new back sight with R.L of the last point obtained from previous set up of the instrument (Heister, et, al., 1999). The arithmetic checks to be applied is given by

$$
\text { B.S. }- \text { F.S. }=\text { LastR.L. }- \text { FirstR.L }
$$

where B.S - Back Sight; - Fore Sight.

\section{X, Y, Z Data of Observed Controls and its Geoidal Heights}

Having obtained the geoidal heights of all the observed controls using Levelling survey methods, it is then possible to have a table containing the coordinates of the observed controls points, their geoidal heights and orthometric heights. Sample Table 1 shows the coordinates of observed controls, their geoidal heights and orthometric heights.

\section{Extracting Orthometric Heights from LIDAR, SRTM and ASTER GDEM}

To facilitate the extraction of orthometric heights from LIDAR, SRTM and ASTER GDEM, it would be necessary to overlay the AutoCAD point's plot of X and Y coordinates of observed control points on each of the data (LIDAR, SRTM and ASTER GDEM). Using spatial analysis tool in an ArcMap environment, we can extract value from points in each of the data. Figures 5, 6 and 7 show the overlay of sample points on each of SRTM, ASTER GDEM and LIDAR respectively (Hofmann-Wellenhof etal, 2008).

\section{Orthometric Heights Extracted From Various Methods}

After extracting all the required orthometric heights from each of the data type used in this study, Table 2 presents the various orthometric heights obtained.

\subsection{Differences between the Orthometric Heights Obtained from Levelling/GPS Survey and Orthometric Heights Obtained by other Methods (SRTM, LIDAR, and ASTER GDEM)}

Finding the differences between the orthometric heights obtained using GPS/Levelling method and that obtained by other methods are necessary to determine how close the heights obtained by other methods to that of Levelling/GPS survey which is considered the most accurate method. The lower the discrepancy, the closer the orthometric heights obtained by that method to that obtained from Levelling/GPS survey method. The higher the discrepancy the further the orthometric heights obtained by that method to that obtained from Levelling/GPS survey method. Sample Table 3 shows the discrepancy between the orthometric heights obtained from Levelling/GPS survey and that obtained from LIDAR, SRTM and ASTER GDEM. 

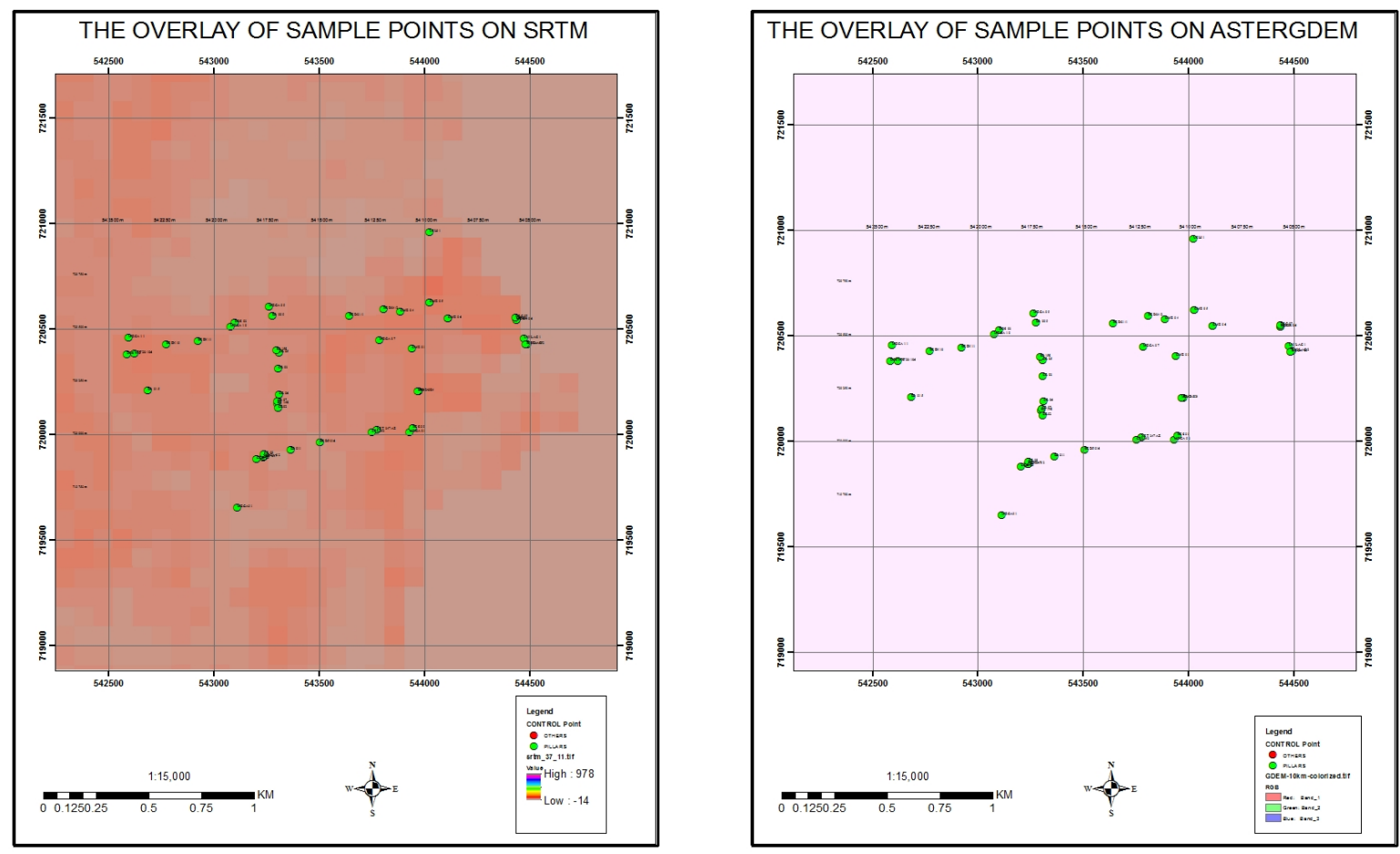

Figure 5: Sample Points Overlaid on SRTM (left).

Figure 6: Sample Points Overlaid On ASTER GDEM (right).

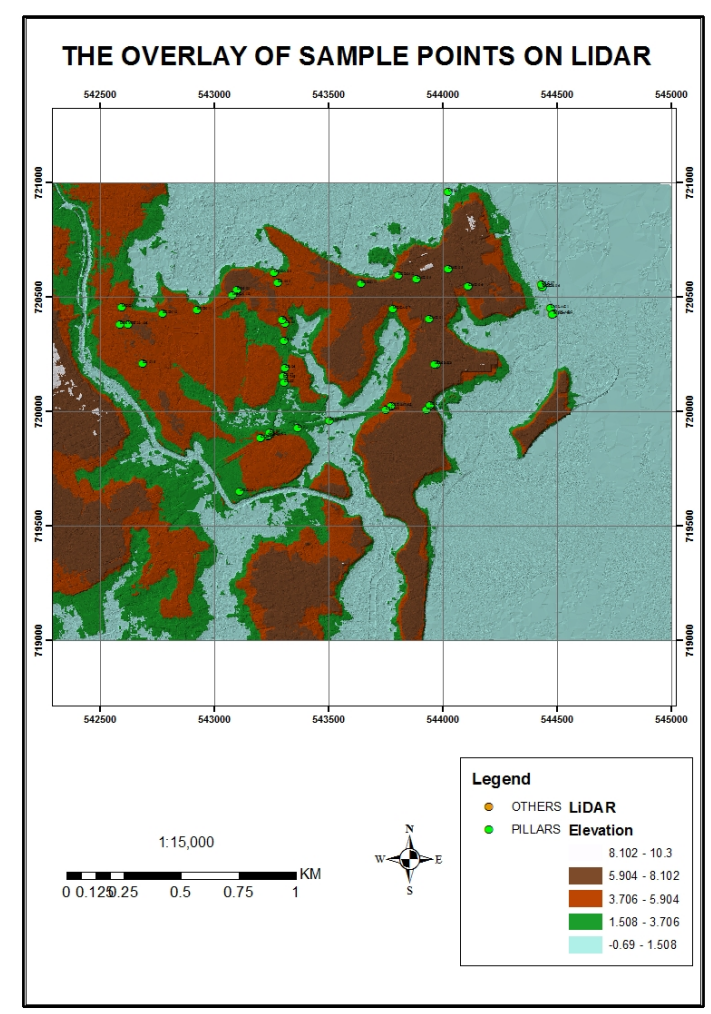

Figure 7: Sample Points Overlaid On LIDAR.. 
Table 3: Percentage Ratio Test

\begin{tabular}{|c|c|c|c|}
\hline Station ID & LIDAR & $\begin{array}{l}\text { of ratio } \\
\text { SRTM }\end{array}$ & ASTER GDEM \\
\hline UNILAG1 & 82 & 0 & 76.02 \\
\hline UNILAG2 & 96 & 0 & 178.08 \\
\hline DSG08 & 50 & 396.51 & 53.86 \\
\hline MEGA05 & 81 & 0 & 139.89 \\
\hline MEGA06 & 87 & 470.15 & 93.79 \\
\hline MEGA03 & 120 & 361.60 & 155.46 \\
\hline PGS09 & 110 & 278.01 & 122.72 \\
\hline GME02 & 106 & 285.71 & 177.89 \\
\hline MEGA04 & 90 & 246.09 & 111.75 \\
\hline GME06 & 127 & 151.75 & 70.95 \\
\hline GME05 & 110 & 238.57 & 138.85 \\
\hline GME04 & 100 & 321.03 & 163.85 \\
\hline PGD84/2 & 120 & 135.21 & 123.99 \\
\hline MEGA07 & 90 & 505.90 & 366.98 \\
\hline PGD81/1 & 115 & 277.22 & 141.18 \\
\hline ED009 & 92 & 220.51 & 147.62 \\
\hline MEGA09 & 89 & 167.79 & 140.20 \\
\hline DOS03 & 117 & 200.53 & 121.06 \\
\hline MEGA10 & 112 & 210.23 & 105.45 \\
\hline MEGA11 & 79 & 251.89 & 108.67 \\
\hline XST347 & 95 & 204.71 & 193.66 \\
\hline MEGA02 & 116 & 170.43 & 17.97 \\
\hline CR08 & 94 & 221.06 & 93.57 \\
\hline SD14S & 77 & 109.29 & 75.94 \\
\hline CR07 & 137 & 170.50 & 127.08 \\
\hline CR06 & 93 & 165.29 & 121.08 \\
\hline CR05 & 84 & 192.36 & 95.12 \\
\hline CR03 & 74 & 126.77 & 79.40 \\
\hline ED011 & 91 & 127.71 & 75.96 \\
\hline DSG07 & 76 & 600.24 & 75.54 \\
\hline CGG/SP & 81 & 211.25 & 172.39 \\
\hline XST347AZ & 83 & 136.22 & 128.62 \\
\hline CBLM3 & 111 & 172.50 & 115.84 \\
\hline YTT28/186 & 121 & 972.45 & 550.61 \\
\hline ED015 & 92 & 221.33 & 113.81 \\
\hline SD15S & 82 & 153.51 & 139.32 \\
\hline DOS14S & 102 & 230.41 & 127.77 \\
\hline GME03 & 135 & 218.47 & 28.10 \\
\hline CR02 & 83 & 164.56 & 94.12 \\
\hline MEGA01 & 90 & 65.98 & 132.85 \\
\hline SGDP016 & 129 & 88.67 & 74.92 \\
\hline SGEN10 & 98 & 223.96 & 87.87 \\
\hline SGEN11 & 121 & 237.15 & 129.08 \\
\hline USL01 & 111 & 50.83 & 116.56 \\
\hline
\end{tabular}


Table 4: Descriptive Statistics Test Result.

\begin{tabular}{|l|c|c|c|c|c|c|c|}
\hline & $\mathrm{N}$ & Range & Minimum & Maximum & \multicolumn{2}{|c|}{ Mean } & Std. Dev. \\
\cline { 2 - 8 } & Statistic & Statistic & Statistic & Statistic & Statistic & Std. Error & Statistic \\
\hline LIDAR ORTHO & 44 & 7 & 1 & 8 & 3.94 & .245 & 1.317 \\
GROUND ORTHO & 44 & 5 & 1 & 6 & 3.96 & .152 & 1.010 \\
STRM ORTHO & 44 & 12 & 0 & 12 & 8.00 & .473 & 3.137 \\
ASTER ORTHO & 44 & 7 & 1 & 8 & 4.17 & .262 & 1.736 \\
Valid N & 44 & & & & & & \\
\hline
\end{tabular}

\subsection{Descriptive Statistics Test on the Acquired Orthometric Heights}

Descriptive statistics is studied to learn how to gather together and summarized data in such a way as to make them readily comprehensive. The result of the descriptive statistics test carried out on the various orthometric heights acquired from all the methods was shown in Table 4.

\subsection{Correlation Test Between Various Orthometric Heights Acquired}

In summary, correlation coefficients are used to assess the strength and direction of the linear relationships between pairs of variables. When both variables are normally distributed, Pearson's correlation coefficient is used, otherwise Spearman's correlation coefficient is required. In statistics, the correlation coefficient $r$ measures the strength and direction of a linear relationship between two variables on a scatterplot. The value of $r$ is always between +1 and -1 . To interpret its value, see which of the following values your correlation $r$ is closest to:

Exactly -1 A perfect downhill (negative) linear relationship

-0.70 A strong downhill (negative) linear relationship

-0.50 A moderate downhill (negative) relationship

-0.30 A weak downhill (negative) linear relationship

$0 \quad$ No linear relationship

$+0.30 \quad$ A weak uphill (positive) linear relationship

+0.50 A moderate uphill (positive) relationship

+0.70 A strong uphill (positive) linear relationship

Exactly $+1 \quad$ A perfect uphill (positive) linear relationship

Correlation is the mutual or degree of relationship between two or more variables i.e. independent and dependent variables. This test was carried out to check the strength of the relationship(s) in the obtained orthometric heights. There are different types of correlation, but for the purpose of this research work the Pearson's correlation method was adopted. For typical variables $\mathrm{X}$ and $\mathrm{Y}$, a positive correlation will be observed when the increasing values of one variable $\mathrm{X}$ are associated with increasing values of the other variable $\mathrm{Y}$. However when an increasing values of one variable $\mathrm{X}$ are associated with decreasing values of the other variable $\mathrm{Y}$ then we have negative correlation.

The product moment correlation coefficient $R$ is given by

$$
R=\frac{N \sum X Y-\sum X \sum Y}{\sqrt{\left(N \sum X^{2}-\left(\sum X\right)^{2}\right)\left(N \sum Y^{2}-\left(\sum Y\right)^{2}\right)}}
$$

Geoinformatics FCE CTU 17(1), 2018 
where $N$ is the number of sample, $X$ and $Y$ are the dependent and the independent variable whose relationship are to be established.

When this correlation test was performed between orthometric heights from Levelling/GPS method and ASTER GDEM method, a low positive correlation of $R=0.179$ was obtained. For correlation analysis between Levelling/GPS method and SRTM method, a week negative correlation of $R=-0.255$. However, when this same test was executed between orthometric heights from Levelling/GPS method and LIDAR method, a strong positive correlation of $R=0.831$ was achieved.

Having carried out the correlation analyses between the orthometric heights generated from ground method and those generated from other methods, Table 5 shows the result of the correlation test.

Table 5: Correlation Analysis between orthometric heights generated from ground method and that generated from other methods.

\begin{tabular}{|c|c|c|c|}
\hline & \multicolumn{3}{|c}{ Correlation R between orthometric heights from levelling/GPS method and } \\
& LIDAR method & ASTER GDEM method & SRTM method \\
\hline Value of R & 0.831 & 0.179 & -0.255 \\
& very high positive & low positive & low negative \\
\hline
\end{tabular}

\subsection{Percentage of Ratio Test}

The percentage ratio test is basically a ratio analysis that compare samples of observation of a given quantity to the corrected value or measure. In this study, each of the orthometric height generated by the ground Levelling/GPS method were taken as the corrected orthometric height for all control points used in this study since the best of height reliability till date is determined by this method, $100 \%$ test value is assigned to heights acquired through this method in relation to height obtained from other methods. Hence, ranks were assigned to the orthometric heights obtained from LIDAR, SRTM and ASTER GDEM data based on the closeness of their orthometric heights to that of the Levelling/GPS method. These ranks are then use to identify the approach that can serve or be adopted as an alternative to orthometric height determination by Levelling/GPS method.

The number of points with orthometric heights value that are close to that of Levelling/GPS method were noted and counted based on the values obtained from their percentage of ratio test. Table 3 shows the percentage of ratio test results and Table 6 presents the number of points in other methods whose orthometric heights are comparably close to the orthometric heights obtained by Levelling/GPS method.

Table 6: The number of points in other methods whose orthometric height is closed to that of Level- ing/GPS method.

\begin{tabular}{|l|c|}
\hline \multicolumn{1}{|c|}{ Method } & Points \\
\hline LIDAR & 26 \\
\hline SRTM & 3 \\
\hline ASTER GDEM & 13 \\
\hline
\end{tabular}




\subsection{One Way ANOVA Test on the Acquired Orthometric Heights}

Analysis of variance is applied after the results of the designed experiment have been obtained. It is used for comparison of more than two means and also to control error due to randomization. Here, measurements are obtained from 4 independent groups of samples, where the number of measurements in each group is uniquely the number of control points used for the study.

Hypothesis formulated include;

Null Hypothesis: There is no difference between the mean weights of all orthometric heights generated.

Alternative Hypothesis: The mean weight of all orthometric heights generated are not equal

$$
H_{1}: \mu_{1} \neq \mu_{2} \neq \mu_{3} \neq \mu_{4}
$$

where are the population mean weight of ground, LIDAR, SRTM and ASTER GDEM orthometric heights respectively.

Table 7 is a highlight of the result of one way ANOVA test carried out on all the acquired orthometric heights.

Table 7: One Way ANOVA Test at 5\% and 1\% Signicance Level

\begin{tabular}{|c|c|c|c|c|c|}
\hline $\begin{array}{c}\text { Variation } \\
\text { source }\end{array}$ & $\begin{array}{c}\text { Degree of } \\
\text { freedom }\end{array}$ & $\begin{array}{c}\text { Mean } \\
\text { square }\end{array}$ & $\begin{array}{c}\mathrm{F} \\
\text { ratio }\end{array}$ & $\begin{array}{c}\text { Significant } \\
\mathrm{t}(1 \%)\end{array}$ & $\begin{array}{c}\text { Significant } \\
\mathrm{t}(5 \%)\end{array}$ \\
\hline $\begin{array}{c}\text { Between } \\
\text { samples }\end{array}$ & 3 & 65.273 & & & \\
\cline { 1 - 3 } $\begin{array}{c}\text { Within } \\
\text { samples }\end{array}$ & 172 & 3.90 & \multirow{2}{*}{16.73} & 3.78 & 2.6 \\
\cline { 1 - 3 } Total & 175 & 4.95 & & & \\
\hline
\end{tabular}

3.6. Profile Plot of 1 to 44 Ground Control Points against their Orthometric Height from Various Methods

Plotting the profile of the orthometric heights obtained from various methods would help us to determine how each of the orthometric height relates with one another. Figure 8 shows the profiles of 1 to 44 ground control points against their orthometric height, obtained from various methods.

\subsection{Result Interpretation from All The Analysis}

Having carried out several analyses on all the orthometric heights acquired from all the methods used, the following results were obtained:

(i) From Table 4 it is obvious that the standard deviations, variances, and standard error increases in the order indicated in the arrow diagram. Levelling/GPS orthometric height $\rightarrow$ LIDAR orthometric $\rightarrow$ ASTER GDEM orthometric $\rightarrow$ SRTM orthometric. This was 


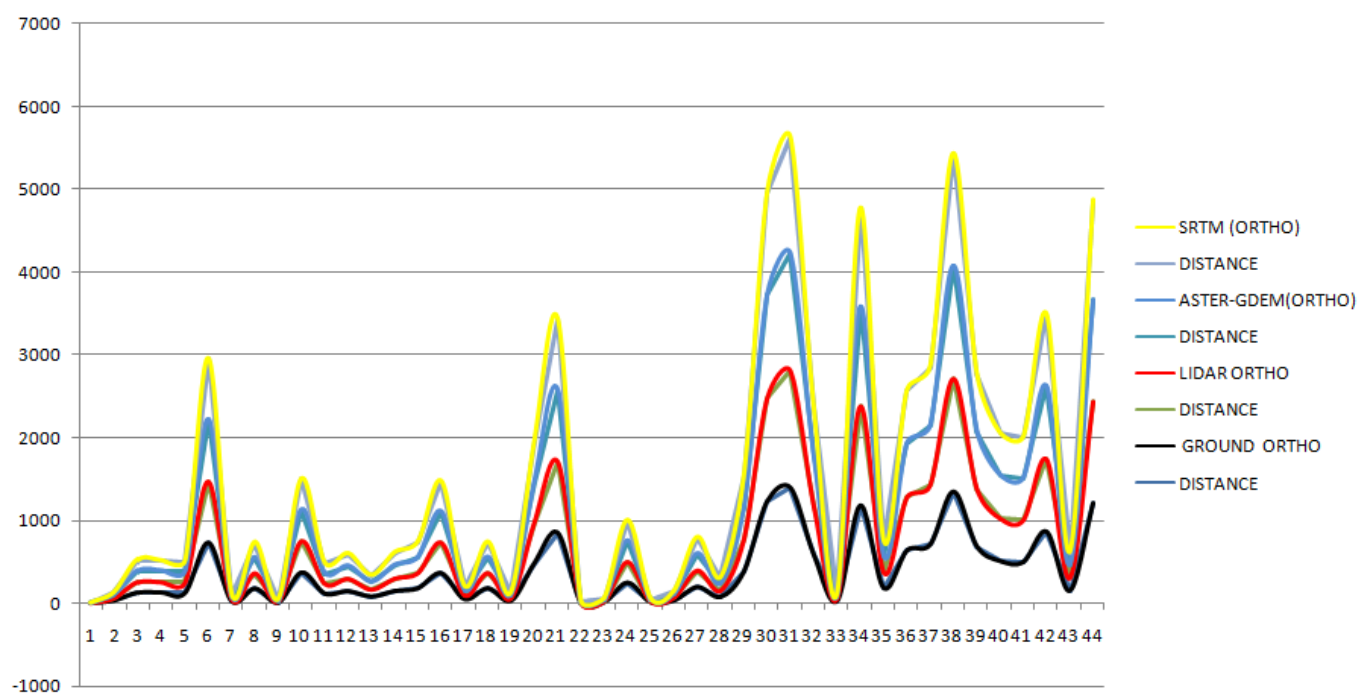

Figure 8: Profiles of 1 to 44 Ground Control Points against their Orthometric Heights obtained from Various Methods.

attributed to differences in the accuracy of the method of data collection and the spatial resolution of the methods used.

(ii) From Table 7, the F tabulated is less than the F calculated (Ftab $<$ Fcal) at $1 \%$ and $5 \%$ level of significance indicating the rejection of null hypothesis thus the mean weight of orthometric heights generated from all the methods are not the same.

(iii) From Table 5, the degree of relationship existing between Levelling/GPS and LIDAR orthometric height was high, indicating the closeness of their orthometric heights at several points. The degree of such relationship is very low between Levelling/GPS and ASTER GDEM orthometric heights; this indicates that the orthometric heights generated by the two methods implies higher disagreement at several points, this can also be attributed to the differences in the accuracy of the method of data collection and the spatial resolution adopted for the two methods. However a negative or inverse relationship exists between Levelling/GPS and SRTM orthometric, indicating a short fall in the degree of estimation of the orthometric heights at those points.

(iv) From Table 6, it is obvious that LIDAR has the highest number points whose orthometric height was closer to that of Levelling/GPS method, this was followed by ASTER GDEM and SRTM has the lowest number of points whose orthometric height was closer to that of Levelling/GPS method.

(v) From the Figure 8 i.e. the profile diagram, there was a likely relationship between the various orthometric heights plotted. This is attributed to the topography of the study area (UNILAG) being in the coastal part of Lagos state with a very low relative orthometric heights.

\section{Conclusion}

From the various analyses carried out, the following are the conclusions inferred: 
(i) From the descriptive statistics test, Table 4, it is obvious that the standard deviations, variances, and standard error increases in the order indicated below. Levelling orthometric height $\rightarrow$ LIDAR orthometric $\rightarrow$ ASTER GDEM orthometric $\rightarrow$ SRTM orthometric. This specifically indicates that the four methods operate at different spatial resolution.

(ii) From the one way ANOVA test, Table 7, the F tabulated is less than the F calculated $($ Ftab $<$ Fcal $)$ at $1 \%$ and $5 \%$ level of significance indicating the rejection of null hypothesis thus the mean weight of orthometric heights generated from all the methods are not the same.

(iii) From the correlation analysis, Table 5, the degree of relationship existing between Levelling/GPS and LIDAR orthometric height was high, indicating the closeness of their orthometric heights at several points. The degree of relationship between Levelling/GPS and ASTER GDEM orthometric heights was very low; this indicates that the orthometric heights generated by the two methods are subject to the differences in the accuracy of the method of data collection and the spatial resolution adopted for the two methods. However a negative or inverse relationship exists between Levelling/GPS and SRTM orthometric height, indicating a short fall in the estimation of these values of the orthometric heights at those points. From this analysis, we inferred that ranking of all these methods can be done with respect to their correlation results, thus it is possible to use orthometric heights generated by LIDAR as an alternative to that generated by Levelling/GPS method, given that all other necessary specification have been satisfied.

(iv) From the percentage of ratio test of Table 3 and the number of points in other methods whose orthometric height is closed to that of Levelling/GPS method Table 6, it is obvious that LIDAR has the highest number points whose orthometric height was closer to that of Levelling/GPS method, this was followed by ASTER GDEM and SRTM has the lowest number of points whose orthometric

(v) It is obvious that ASTER GDEM method is only effective in area with constant cloud cover, SRTM is effective in topographically steep area, and LIDAR is effective for all terrain.

(vi) The smaller extent of missing data in ASTER GDEM than in SRTM is an advantage for the former. This is particularly true in high mountainous areas with steep slopes, where SRTM-3 typically has many missing data. Although ASTER GDEM includes a relatively large amount of missing data in lowlands, below an elevation of $100 \mathrm{~m}$, it is still only about one-half that of the SRTM. Moreover, the amount of data missing in ASTER GDEM should decrease, because the acquisition of new ASTER images is continuing, (Hayakawa, 2008).

(vii) ASTER GDEM has better topographic representation of low-altitude hilly lands, for which SRTM tends to overestimate the height of valley floors, (Deng et al., 2007; http://hydrosheds.cr.usgs.gov/).

(viii) Within airborne context, LiDAR produces high accuracy datasets and are able to collect terrain data over large areas (Leon et al., 2014). Although accurate, the availability of LiDAR data for some regions may be limited due to relatively higher cost plus time constraint. 


\section{References}

[1] Oscar L. Colombo. "Ephemeris errors of GPS satellites". In: 60 (1986), pp. 64-84. ISSN: 0007-4632. DOI: $10.1007 /$ bf 02519355 .

[2] Oscar L. Colombo. "The dynamics of Global Positioning System orbits and the determination of precise ephemerides". In: Journal of Geophysical Research: Solid Earth 94.B7 (1989), pp. 9167-9182. DOI: 10.1029/jb094ib07p09167.

[3] Heister H. et al. "Determination of an orthometric height profile in the Okavango Delta using GPS Levelling". In: Proceedings of the FIG working Week and Survey 1999. TS 20, B1-B10. FIG. Sun City, South Africa, 1999.

[4] Yuichi S. Hayakawa, Takashi Oguchi, and Zhou Lin. "Comparison of new and existing global digital elevation models: ASTER G-DEM and SRTM-3". In: Geophysical Research Letters 35.17 (Sept. 2008). DOI: 10.1029/2008g1035036.

[5] Bernhard Hofmann-Wellenhof, Herbert Lichtenegger, and Elmar Wasle. GNSS - Global Navigation Satellite Systems. Springer, 2008. DOI: 10.1007/978-3-211-73017-1.

[6] Javier X. Leon, Gerard B. M. Heuvelink, and Stuart R. Phinn. "Incorporating DEM Uncertainty in Coastal Inundation Mapping". In: PLoS ONE 9.9 (Sept. 2014). Ed. by Guy J.-P. Schumann, e108727. DOI: 10.1371/journal.pone.0108727.

[7] E. Rodríguez et al. An assessment of the SRTM topographic products. Tech. rep. D-31639. Jet Propulsion Laboratory, Pasadena, California: NASA, 2005. URL: https ://www2.jpl. nasa.gov/srtm/SRTM_D31639.pdf. 